\title{
Effect of Sodium Alginate in Combination with Zataria multiflora Boiss. on Phenolic Compounds, Antioxidant Activity, and Browning Enzymes of Fresh In-Hull Pistachio (Pistacia vera L.)
}

\author{
Maryam Hashemi (D), ${ }^{1,2}$ Ahmad Shakerardekani (D), ${ }^{2}$ Abdolmajid Mirzaalian Dastjerdi (D), \\ and SeyedHossein Mirdehghan $\mathbb{D D}^{3}$ \\ ${ }^{1}$ Departmentof Horticultural Sciences, University of Hormozgan, Bandar Abbas, Iran \\ ${ }^{2}$ Pistachio Research Center, Horticultural Sciences Research Institute, Agricultural Research, \\ Education and Extension Organization (AREEO), Rafsanjan, Iran \\ ${ }^{3}$ Departmentof Horticultural Sciences, Vali-e-Asr University of Rafsanjan, Iran
}

Correspondence should be addressed to Maryam Hashemi; mhashemi63@yahoo.com

Received 10 July 2021; Revised 29 August 2021; Accepted 25 September 2021; Published 7 October 2021

Academic Editor: Barbara Speranza

Copyright (c) 2021 Maryam Hashemi et al. This is an open access article distributed under the Creative Commons Attribution License, which permits unrestricted use, distribution, and reproduction in any medium, provided the original work is properly cited.

\begin{abstract}
The color of fresh pistachio is used as a postharvest quality indicator. The present study was performed to investigate the chemical properties of fresh pistachios coated with different sodium alginate concentrations ( 1 and 1.5\%), various amounts of Shirazi thyme essential oil ( 0.3 and $0.5 \%)$, and their combination during storage $\left(2 \pm 1^{\circ} \mathrm{C}\right.$ and $\left.85 \pm 5 \% \mathrm{RH}\right)$. Over the storage duration, chemical parameters were measured on days 13,26 , and 39 . The results showed that although the application of sodium alginate in combination with thyme essential oil decreased polyphenol oxidase activity in comparison with other treatments, the highest total phenolics and phenylalanine ammonia lyase activity were found in pistachios coated with alginate (1\%) + thyme essential oil ( $0.3 \%$ and $0.5 \%)$. In general, it was proven that treatments containing $1 \%$ alginate $+0.3 \%$ essential oil had the ability to maintain the quality of fresh pistachio fruit approximately over 39 days of storage.
\end{abstract}

\section{Introduction}

Pistachio nuts contain considerable amounts of essential minerals, phenolics, essential fatty acids, proteins, and vitamin A [1] and are known as healthy food. Pistachio is commonly used in the form of a dry product as nut, but the consumption of fresh pistachio has become more popular in the last decades [2].

Weight loss, lipid oxidation, browning, and microbial growth of fresh pistachio play important roles in its final appearance and quality during marketing [3]. Studies have shown that, after harvesting fresh pistachio, it immediately becomes brown and decayed that affect its final market acceptability [4]. Browning is one of the most important phenomena that occurs in the pistachio hull during processing and storage [3]. It is a common color change among fresh vegetables and fruits and takes place because of the reaction of phenolic compounds, oxygen, and polyphenol oxidase (PPO) enzyme. Biochemical studies have shown that the oxidation of polyphenols (mainly the conversion of ortho-diphenols into quinones and semiquinones) is the reason for hull browning [4].

Edible coating of fresh products is applied to avoid quality deterioration of fresh fruits [5]. It can control gas exchange from the fruit surface, decrease respiration rate, and control water evaporation from fruits, thereby reducing moisture loss [6]. Furthermore, edible coating technology improves product quality along with its shelf life by changing the internal atmosphere and reducing microbial proliferation [7]. However, choosing suitable formulations for edible coatings considerably affects their effectiveness. Azarakhsh et al. [8] reported that respiration rate and weight loss were 
decreased in alginate-coated pineapples compared with control fruits. Ribeiro et al. [9] showed that alginate was helpful in delaying color change, weight loss, and softening of coated plums. They concluded that edible coatings acted as barriers on the surface of fruit and reduced its permeability to gases $\left(\mathrm{CO}_{2}\right.$ and $\left.\mathrm{O}_{2}\right)$ and water vapor. This decreased the rate of respiration and transpiration and delayed the ripening process.

The efficacy of edible coating could be improved by the addition of antibrowning and antimicrobial agents [10]. Nowadays, functional additives such as essential oils (EOs) are mainly added to edible coating formulations to enhance the appearance, integrity, microbial safety, and mechanical strength of food [11].

Direct surface application of antifungal and antibacterial compounds has slight benefits because active ingredients are neutralized at contact or are diffused into the bulk of fruit. Therefore, the addition of EOs into edible coating formulations is an efficient strategy to improve the functionality of coatings [12].

Shirazi thyme (Zataria multiflora Boiss.) has a high percentage of essential oil with antibacterial and antifungal activities. Application of Zataria multiflora essential oil in various antimicrobial edible coatings including alginate $[13,14]$, gum arabic [15], chitosan [16], and carboxymethyl cellulose [17] showed to increase total phenolic compounds and, as a result, antioxidant activity of films. The results showed that films containing essential oil could be potentially applied in active biodegradable packaging materials to inhibit or delay deterioration and oxidation and prevent microbial activity [17].

Considering limited studies on fresh pistachio storability and the potential of sodium alginate and Z. multiflora essential oil to prevent the activity of PPO and oxidation of phenolics, treatment with sodium alginate and $Z$. multiflora essential oil was applied to decrease browning. The present study aimed to investigate the possible potentials of various formulations of sodium alginate and Z. multiflora essential oil and their combination for suppressing the browning of fresh pistachio fruits.

\section{Materials and Methods}

2.1. Materials. Fresh pistachio (Pistacia vera L.) fruits cv. 'Ahmad-Aghaei' were harvested manually on October 10 from 30-year-old pistachio trees. They were instantly transported to the laboratory of postharvest, and those without crack and with uniform size, color, and shape were separated from the clusters.

Food grade sodium alginate, glycerol, and sunflower oil were obtained from Sigma-Aldrich Chemicals (Germany). Zataria multiflora essential oil was purchased from Barij Essence Company (Kashan, Iran).

2.2. Edible Coating Solutions. Sodium alginate edible coating was prepared according to the method reported by Azarakhsh et al. [18]. To prepare 1 and $1.5 \%(w / v)$ edible coatings, 1 and $1.5 \mathrm{~g}$ sodium alginate powder (Merck, Germany) were dispersed in $100 \mathrm{ml}$ distilled water, respectively. The solutions were heated at $70^{\circ} \mathrm{C}$, and stirring was carried out until the clear appearance of the mixture. Then, $1.5 \%(\mathrm{w} / \mathrm{v})$ glycerol was added followed by $0.025 \%(\mathrm{w} / \mathrm{v})$ sunflower oil. Then, Zataria multiflora Boiss. EO at concentrations 0\% (control), $0.3 \%$, and $0.5 \%(\mathrm{w} / \mathrm{v})$ was added. Finally, these mixtures were homogenized for $5 \mathrm{~min}$.

2.3. Edible Coating Treatments and Storage. Edible coatings were applied according to the method reported by Azarakhsh et al. [18]. The fruits were immersed in coating solutions for $3 \mathrm{~min}$ and then air-dried for $1 \mathrm{~h}$ at $25 \pm 2^{\circ} \mathrm{C}$. Then, they were immersed in calcium chloride $(2 \% \mathrm{w} / \mathrm{v})$ for $2 \mathrm{~min}$ to allow gel formation and air-dried at $25 \pm 2{ }^{\circ} \mathrm{C}$. $200-250 \mathrm{~g}$ pistachio fruit was placed in each polypropylene container with dimensions $12 \times 15.5 \times 10.5 \mathrm{~cm}$. Coated pistachios were stored at $2 \pm 1^{\circ} \mathrm{C}$ and $85 \pm 5 \%$ RH for 39 days. Fresh pistachio characteristics were determined every 13 days during storage.

2.4. Total Phenolics (TPs). TPs were measured by the Folin-Ciocalteu reagent [19]. $0.5 \mathrm{~g}$ flesh tissue of the pistachio hull was homogenized with $3 \mathrm{ml}$ methanol (85\%) and then centrifuged at $12,000 \times \mathrm{g}$ for $20 \mathrm{~min}$ at $4^{\circ} \mathrm{C}$ to remove any undesirable impurities. The supernatant was separated and used as the extract. $300 \mu \mathrm{L}$ extract was mixed with $1200 \mu \mathrm{L} \mathrm{7 \%}$ sodium carbonate and maintained at room temperature for $5 \mathrm{~min}$. Then, $1500 \mu \mathrm{L} \mathrm{10 \%} \mathrm{Folin-Ciocalteu}$ reagent was added, and the mixture was shaken for $90 \mathrm{~min}$ in darkness at room temperature. Absorbance was read at $760 \mathrm{~nm}$ by using a spectrophotometer (Epoch Biotech, Germany). Gallic acid was used for drawing the standard curve. The concentration of the total phenolic content was expressed as mg gallic acid equivalents per $100 \mathrm{~g}$ FW.

2.5. DPPH Radical Scavenging Activity. Antioxidant activity (AA) was measured according to the 2,2-diphenyl-1-picrylhydrazyl (DPPH) radical scavenging method described by Brand-Williams et al. [20]. $0.1 \mathrm{ml}$ fruit hull extract, $1 \mathrm{ml}$ DPPH (0.1 mM), and $1 \mathrm{ml}$ Tris- $\mathrm{HCl}(\mathrm{pH} 7.5)$ buffer were mixed using a vortex. The final mixture was incubated for $30 \mathrm{~min}$ in a dark room. Then, mixture absorbance was read at $517 \mathrm{~nm}$ by using a spectrophotometer (Epoch Biotech, Germany), and AA percentage was calculated using the following equation:

$$
\mathrm{AA}(\%)=\left[1-\frac{\text { Asample }}{\text { Acontrol }}\right] \times 100 \text {. }
$$

2.6. Enzyme Activities. $0.5 \mathrm{~g}$ frozen fresh fruit hull was powdered in a chilled pestle using liquid nitrogen and homogenized in $1 \mathrm{~mL} 100 \mathrm{mM}$ phosphate buffer ( $\mathrm{pH} \mathrm{7)}$ containing $0.5 \mathrm{mM}$ EDTA and $6 \% \quad(\mathrm{w} / \mathrm{v})$ polyvinylpolypyrrolidone (PVPP). The mixture was centrifuged at $15,000 \times \mathrm{g}$ for $20 \mathrm{~min}$ at $4^{\circ} \mathrm{C}$, and the supernatant was used for the measurement of enzyme activities. 
2.7. Polyphenol Oxidase (PPO) Activity Assay. Polyphenol oxidase (PPO) activity was determined using the methods proposed by Koushesh Saba et al. [21]. Aliquots of the supernatant were added to two solutions containing catechol and pyrogallol at concentrations of $0.05 \mathrm{M}$ and $0.02 \mathrm{M}$, respectively. Absorbance increase was monitored at $420 \mathrm{~nm}$ for $1 \mathrm{~min}$ at room temperature, and enzyme activity was expressed as unit per gram of fresh weight per minute.

2.8. Phenylalanine Ammonia Lyase (PAL) Activity Assay. For the measurement of phenylalanine ammonia lyase (PAL) activity, $0.1 \mathrm{~mL}$ supernatant was added to a mixture containing $0.5 \mathrm{~mL} 10 \mathrm{mM}$ L-phenylalanine, $1 \mathrm{~mL} 50 \mathrm{mM}$ phosphate buffer ( $\mathrm{pH} 7)$, and $0.4 \mathrm{ml}$ double-distilled water. After mixture incubation at $40^{\circ} \mathrm{C}$ for $1 \mathrm{~h}$, the reaction was stopped by adding $0.5 \mathrm{~mL} 6 \mathrm{mM} \mathrm{HCl}$. PAL activity was measured by the absorbance of the mixture at $290 \mathrm{~nm}$ based on the production of cinnamic acid. PAL enzyme activity was expressed as mg of cinnamic acid per gram of fresh weight per min [22].

2.9. Statistical Analysis. The experiment was set as factorial based on randomized complete block design with nine treatments and three replications. The source of variation included edible coatings, essential oils, and storage duration. Analysis of variance (ANOVA) was performed using SAS software (version 9.1). The mean values were compared according to Duncan's multiple range test at $P<0.05$.

\section{Results and Discussion}

3.1. Total Phenolic Concentration and DPPH Radical Scavenging Activity. The effects of edible coating, essential oil, and essential oil-enriched edible coatings on the TP of the fresh hull are illustrated in Figure 1(a). Among different treatments, fruits covered with $1 \%$ sodium alginate $+0.3 \%$ thyme showed the highest TP content $(57.01 \mathrm{mg} / 100 \mathrm{~g}$ fresh weight) after 39 days of storage. However, fruits treated with $0.5 \%$ thyme essential oil showed the lowest TP content $(49.91 \mathrm{mg} / 100 \mathrm{~g}$ fresh weight) among all treatments (Figure 1(a)). As shown in Figure 1(b), initial DPPH radical scavenging activity of the fresh pistachio hull was $39.77 \%$. During the storage, DPPH radical scavenging activities of all treated and control fruits were dramatically decreased and reached the lowest level after 13 days of storage. The DPPH radical scavenging level started to increase with increasing storage period up to 26 days and then decreased at the end of storage, but it was higher in all storage times in treated fresh pistachios than control ones (Figure 1(b)).

The reduction of antioxidant capacity in fruits could be attributed to fruit senescence and higher respiration rate due to the degradation and loss of some phenolic compounds. Hashemi et al. [13] showed that sodium alginate edible coating enriched with Zataria multiflora essential oil increased phenolic compounds in the kernels of coated fresh pistachio fruits. Other studies have shown that the application of Zataria multiflora Boiss. essential oil-enriched gum arabic coated on fresh pistachio resulted in higher TP compared to control fruits [15]. Ali et al. stated decreased phenolic content is a natural part of senescence which can also be due to cell wall degradation when fruit is stored over long periods [23].

Hashemi et al. [24] reported that apricots coated with basil seed gum (BSG) + Origanum vulgare essential oil (2-6\%) showed the highest antioxidant activity, while control and BSG-coated samples gave the lowest amount of antioxidants at the end of storage time. These effects could be the result of the oxygen barrier properties of edible coatings and the capacity of essential oil components to retain fruit quality factors along with the inhibition of enzyme activity that degrades antioxidant compounds [25]. Thus, the increase of antioxidant capacity is related to the increase in the TP content. Oms-Oliu et al. [26] observed that the increase of phenolic compounds increased the antioxidant capacity of 'Piel de Sapo melon during 14 days of storage period at $4^{\circ} \mathrm{C}$. Our results clearly revealed the increment of $\mathrm{TP}$ compounds of fruits coated with alginate + thyme oil. Similarly, thyme oil added to packaging materials increased the amount of TPs and flavonoids (catechin) in avocado fruits [27]. A similar trend was also stated by Ali et al. [28] for tomato fruits coated with gum arabic (5\%) edible coatings. However, the increase of the total phenolic content might also increase due to the essential oil component in basil seed gum coating, which could delay the oxidation processes of phenolic compounds by scavenging free radicals and oxygen [29].

3.2. PPO and PAL Activity. As shown in Figures 2(a) and 2(b), the activity of enzymes associated with the browning of the fresh hull, i.e., PAL and PPO enzymes, was significantly affected by edible coating formulations. The activity of the PPO enzyme in fresh hulls was increased during storage so that the highest activity of PPO was observed in control (uncoated) pistachios and those coated with 1.5\% sodium alginate $+0.5 \%$ essential oil after 39 days of storage, while the lowest levels were obtained in fruits treated with $1 \%$ sodium alginate solution $+0.3 \%$ thyme essential oil and $0.3 \%$ essential oil (Figure 2(a)).

According to Figure 2(b), PAL enzyme activity in treated and control fruits was increased during the storage period. The lowest activity of the PAL enzyme was observed in control fruits. The fruit samples treated with $1 \%$ sodium alginate $+0.3 \%$ essential oil showed the highest level of PAL activity; however, it had no significant differences with other treatments except for $1.5 \%$ sodium alginate $+0.5 \%$ essential oil (Figure 2(b)).

PPO is a key enzyme in the texture browning of fruits and vegetables. Some assessments reported a significant increase in PPO activity during storage [30]. Enzymatic browning is often accompanied with damage to the cell membrane in texture [31]. When the integrity of the membrane is reduced, phenolic compounds are exposed to oxygen and oxidized by the catalytic activity of the released polyphenol oxidase [31]. Kader [32] reported that enzymatic browning can be reduced at low levels of oxygen. An 


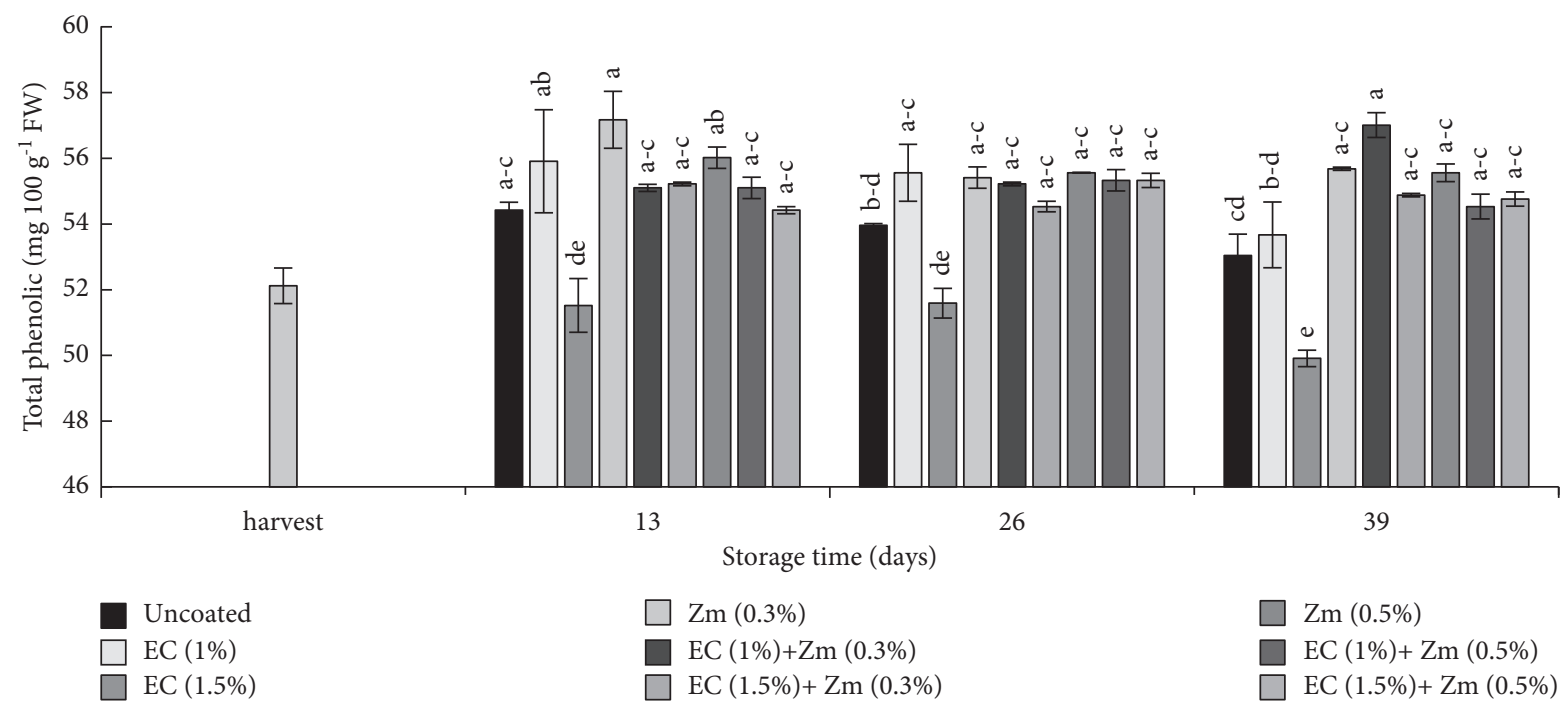

(a)


(b)

Figure 1: Total phenolics (a) of the fresh pistachio hull coated with sodium alginate + Zataria multiflora and DPPH radical scavenging activity (b) in the fresh pistachio hull treated with Zataria multiflora during storage at $3 \pm 1^{\circ} \mathrm{C}$. Means $(n=3)$ with the same letters are not significantly different according to Duncan's test $(p<0.05)$. EC: alginate-based edible coating; Zm: Zataria multiflora.

increase of PPO activity was observed in pistachio during storage, and as a result, the oxidization of phenolic compounds occurring during this process led to lower amounts of polyphenol compounds [33]. In contrast, higher phenolic contents of samples coated with $1 \%$ alginate $+0.3 \%$ thyme might be attributed to lower PPO enzyme activities. It seemed that alginate in solutions acted as a barrier for oxygen needed for PPO activity. In addition, de Sousa [34] reported that mushrooms coated with alginate enriched with eugenol essential oil and cinnamic acid reduced polyphenol oxidase activity which resulted in lower enzymatic browning, and alginate coating formed a protective barrier on the surface of samples, reducing the supply of $\mathrm{O}_{2}$ which can help reduce PPO activity. Previous studies have shown that the application of Arabic gum and sodium casein in combination with cinnamon and lemongrass essential oils on guava [11] was effective in reducing the activity of the PPO enzyme. A previous study examining the effects of gum arabic in combination with Zataria multiflora essential oil on fresh pistachio fruits also showed that these treatments were effective in reducing PPO activity [15]. Regarding the PAL enzyme, several studies have shown that the accumulation of phenols and anthocyanins was correlated with the increase of the activity of this enzyme in some fruits. This is the first key enzyme involved in the biosynthesis of phenols in fruits and vegetables [35]. In addition, herbal essences have not only antimicrobial properties but also the ability to increase TP compounds [36]. This may be because essential oils play positive roles in secondary plant metabolites and stimulate the biosynthesis of phenolic compounds and anthocyanins by inducing an increase in the activity of this enzyme [37]. In addition, Chiabrando and Giacalone 


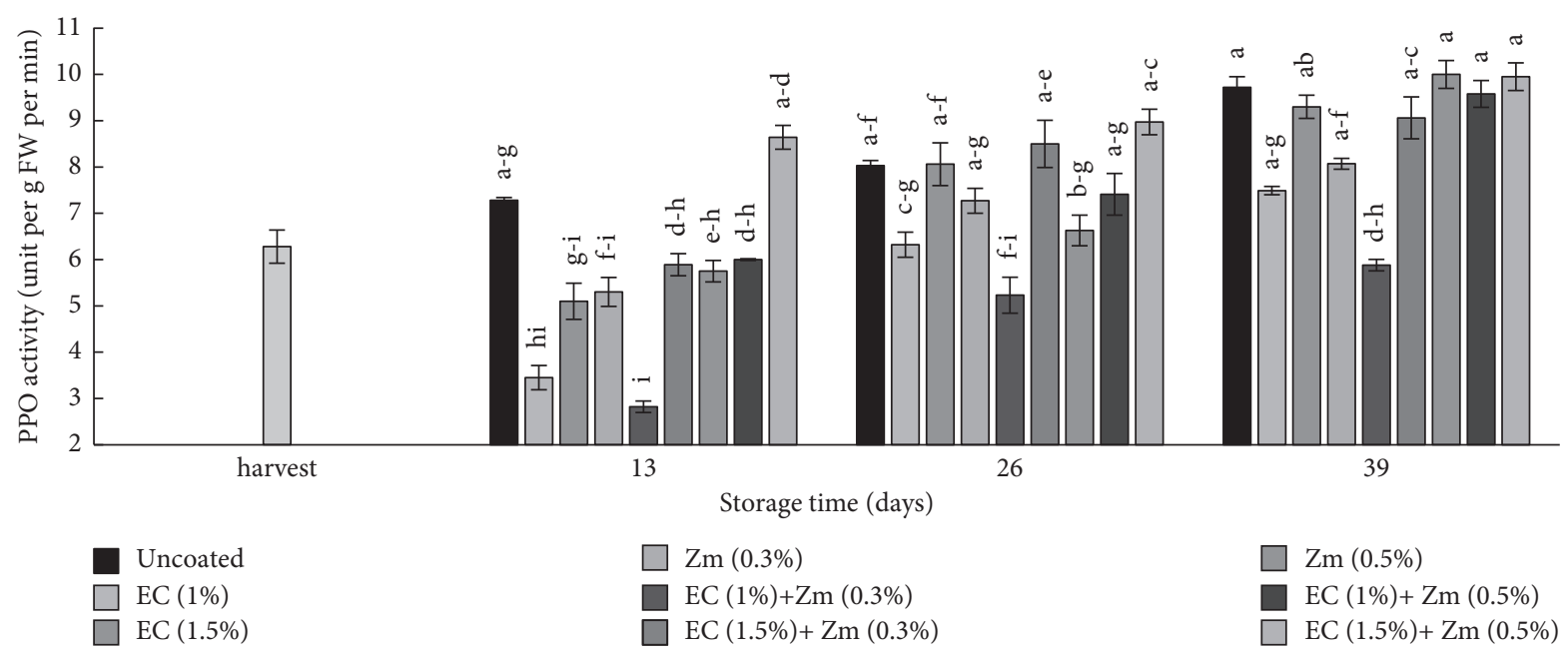

(a)

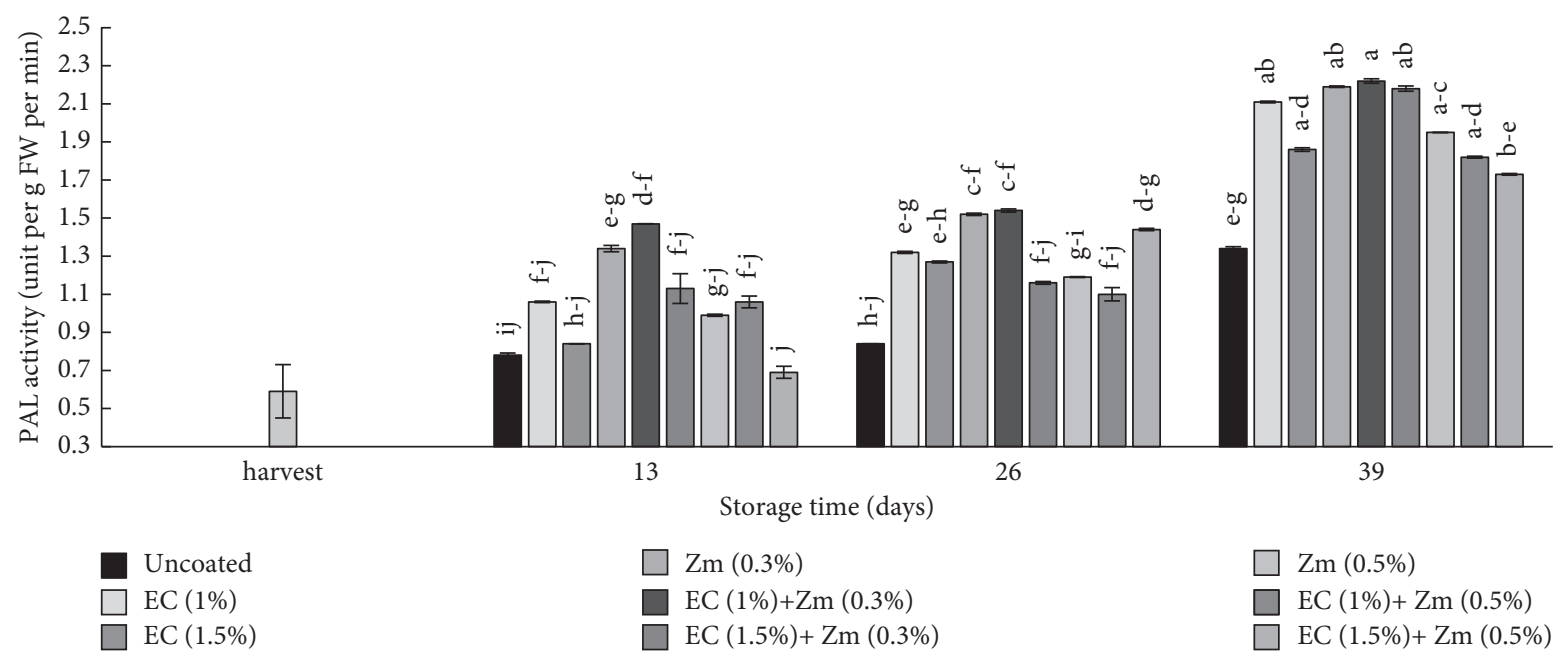

(b)

FIGURe 2: PPO (a) and PAL activity (b) of the fresh pistachio hull coated with sodium alginate + Zataria multiflora at $3 \pm 1^{\circ} \mathrm{C}$. Means $(n=3)$ with the same letters are not significantly different according to Duncan's test $(p>0.05)$. EC: alginate-based edible coating; Zm: Zataria multiflora.

[34] reported that fresh-cut apple coated with alginate enriched with cinnamon and rosemary essential oils reduced polyphenol oxidase activity which resulted in lower enzymatic browning and alginate coating formed a protective barrier on the surface of samples, reducing the supply of $\mathrm{O} 2$ which can help reducing PPO activity. ${ }^{*}$ should replace the previous text.

\section{Conclusion}

One of the most important issues associated with the storage of fresh pistachios is their short storage life. Fresh pistachios lose their quality shortly after harvest because the pistachio hull is very perishable and is rapidly spoiled and browned. Application of edible coatings is an important protection method. However, this method alone is not enough. Certain functional and bioactive compounds can be incorporated into edible coatings, thus enhancing the safety of coated products and bringing benefits to the health of consumers. Thus, edible coatings based on the incorporation of active ingredients, especially essential oils, are considered as one of the approaches with the greatest interest for managing the quality of fresh products. Such a strategy can inhibit enzymatic or biochemical damage during postharvest storage. This study showed that the application of alginate coating enriched with Zataria multiflora could reduce the browning of fresh pistachios during storage. Based on our findings, control (uncoated) fruits showed the most intense hull browning followed by samples treated with $0.5 \%$ essential oil, but it should be noted that careful selection of thyme concentration was required in coating formulations to improve the storability of fresh in-hull pistachio fruits. Also, the lowest PPO enzyme activity was observed in the samples coated with $1 \%$ sodium alginate $+0.3 \%$ Zataria multiflora essential oil. In addition, a higher TP content in the hull was also obtained in fruits coated with $1 \%$ sodium alginate $+0.3 \%$ Zataria multiflora essential oil which was selected as the best formulation for increasing the storage life of fresh pistachio. 


\section{Data Availability}

The data belong to a project, and the authors do not have the permission to publish and/or share the data and provide appropriate attribution.

\section{Conflicts of Interest}

The authors declare no conflicts of interest.

\section{Acknowledgments}

This research was supported by the University of Hormozgan and the Pistachio Research Center of Rafsanjan.

\section{References}

[1] I. Ozturk, O. Sagdic, H. Yalcin, T. D. Capar, and M. H. Asyali, "The effects of packaging type on the quality characteristics of fresh raw pistachios (Pistacia vera L.) during the storage," LWT-Food Science and Technology, vol. 65, pp. 457-463, 2016.

[2] A. Sheikhi, S. H. Mirdehghan, and L. Ferguson, "Extending storage potential of de-hulled fresh pistachios in passivemodified atmosphere," Journal of the Science of Food and Agriculture, vol. 99, no. 7, pp. 3426-3433, 2019.

[3] H. Tavakolipour and A. Kalbasi-Ashtari, "Estimation of moisture sorption isotherms in Kerman pistachio nuts," Journal of Food Process Engineering, vol. 31, no. 4, pp. 564582, 2008.

[4] S. Gheysarbigi, S. H. Mirdehghan, M. Ghasemnezhad, and F. Nazoori, "The inhibitory effect of nitric oxide on enzymatic browning reactions of in-package fresh pistachios(Pistacia vera L.)," Postharvest Biology and Technology, vol. 159, Article ID 110998, 2020.

[5] J. Synowiecki and N. A. Al-Khateeb, "Production, properties, and some new applications of chitin and its derivatives," Critical Reviews in Food Science and Nutrition, vol. 43, no. 2, pp. 145-171, 2003.

[6] J. Duan, R. Wu, B. C. Strik, and Y. Zhao, "Effect of edible coatings on the quality of fresh blueberries (duke and elliott) under commercial storage conditions," Postharvest Biology and Technology, vol. 59, no. 1, pp. 71-79, 2011.

[7] S. Benítez, I. Achaerandio, M. Pujolà, and F. Sepulcre, "Aloe vera as an alternative to traditional edible coatings used in fresh-cut fruits: a case of study with kiwifruit slices," LWTFood Science and Technology, vol. 61, no. 1, pp. 184-193, 2015.

[8] N. Azarakhsh, A. Osman, H. M. Ghazali, C. P. Tan, and N. MohdAdzahan, "Optimization of alginate and gellan-based edible coating formulations for fresh-cut pineapples," International Food Research Journal, vol. 19, pp. 279-285, 2012.

[9] C. Ribeiro, A. A. Vicente, J. A. Teixeira, and C. Miranda, "Optimization of edible coating composition to retard strawberry fruit senescence," Postharvest Biology and Technology, vol. 44, no. 1, pp. 63-70, 2007.

[10] M. A. Rojas-Graü, R. Soliva-Fortuny, and O. Martín-Belloso, "Edible coatings to incorporate active ingredients to fresh-cut fruits: a review," Trends in Food Science \& Technology, vol. 20, no. 10, pp. 438-447, 2009.

[11] S. B. Murmu and H. N. Mishra, "Optimization of the Arabic gum based edible coating formulations with sodium caseinate and tulsi extract for guava," LWT-Food Science and Technology, vol. 80, pp. 271-279, 2017.

[12] L. Sánchez-González, M. Vargas, C. González-Martínez, A. Chiralt, and M. Cháfer, "Use of essential oils in bioactive edible coatings: a review," Food Engineering Reviews, vol. 3, no. 1, pp. 1-16, 2011.

[13] M. Hashemi, A. M. Dastjerdi, A. Shakerardekani, and S. H. Mirdehghan, "Effect of alginate coating enriched with shirazi thyme essential oil on quality of the fresh pistachio (Pistacia vera L.)," Journal of Food Science and TechnologyMysore, vol. 58, 2020.

[14] A. Shakerardekani, M. Hashemi, M. Shahedi, and A. MirzaalianDastjerdi, "Enhancing the quality of fresh pistachio fruit using sodium alginate enriched with thyme essential oil," Journal of Agricultural Science and Technology, vol. 23, no. 1, pp. 65-82, 2021.

[15] M. Hashemi, A. M. Dastjerdi, S. H. Mirdehghan, A. Shakerardekani, and J. B. Golding, "Incorporation of Zataria multiflora boiss essential oil into gum Arabic edible coating to maintain the quality properties of fresh in-hull pistachio (Pistacia vera L.)," Food Packaging and Shelf life, vol. 30, p. 100724, 2021.

[16] M. Moradi, H. Tajik, S. M. Razavi Rohani et al., "Characterization of antioxidant chitosan film incorporated with Zataria multiflora boiss essential oil and grape seed extract," LWT- Food Science and Technology, vol. 46, no. 2, pp. 477484, 2012.

[17] A. Dashipour, V. Razavilar, H. Hosseini et al., "Antioxidant and antimicrobial carboxymethyl cellulose films containing Zataria multiflora essential oil," International Journal of Biological Macromolecules, vol. 72, pp. 606-613, 2015.

[18] N. Azarakhsh, A. Osman, H. M. Ghazali, C. P. Tan, and N. Mohd Adzahan, "Lemongrass essential oil incorporated into alginate-based edible coating for shelf-life extension and quality retention of fresh-cut pineapple," Postharvest Biology and Technology, vol. 88, pp. 1-7, 2014.

[19] V. L. Singelton and J. A. Rossi, "Colorimetry of total phenolics with phosphomolybdic-phosphotungstic acid reagents," American Journal of Enology and Viticulture, vol. 16, no. 39, pp. 144-158, 1965.

[20] W. Brand-Williams, M. E. Cuvelier, and C. Berset, "Use of a free radical method to evaluate antioxidant activity," $L W T$ Food Science and Technology, vol. 28, no. 1, pp. 25-30, 1995.

[21] M. Koushesh Saba, K. Arzani, and M. Barzegar, "Postharvest polyamine application alleviates chilling injury and affects apricot storage ability," Journal of Agricultural and Food Chemistry, vol. 60, no. 36, pp. 8947-8953, 2012.

[22] T. Nguyen, S. Ketsa, and W. G. van Doorn, "Relationship between browning and the activities of polyphenoloxidase and phenylalanine ammonia lyase in banana peel during low temperature storage," Postharvest Biology and Technology, vol. 30, no. 2, pp. 187-193, 2003.

[23] A. Ali, M. Maqbool, S. Ramachandran, and P. G. Alderson, "Gum Arabic as a novel edible coating for enhancing shelf-life and improving postharvest quality of tomato (Solanum lycopersicum L.) fruit," Postharvest Biology and Technology, vol. 58 , no. 1 , pp. 42-47, 2010.

[24] S. M. B. Hashemi, A. Mousavi Khaneghah, M. Ghaderi Ghahfarrokhi, and I. Eş, "Basil-seed gum containing Origanum vulgare subsp. viride essential oil as edible coating for fresh cut apricots," Postharvest Biology and Technology, vol. 125, pp. 26-34, 2017.

[25] O. B. Sogvar, M. Koushesh Saba, and A. Emamifar, "Aloe vera and ascorbic acid coatings maintain postharvest quality and reduce microbial load of strawberry fruit," Postharvest Biology and Technology, vol. 114, pp. 29-35, 2016.

[26] G. Oms-Oliu, R. Soliva-Fortuny, and O. Martín-Belloso, "Using polysaccharide-based edible coatings to enhance 
quality and antioxidant properties of fresh-cut melon," $L W T$ Food Science and Technology, vol. 41, no. 10, pp. 1862-1870, 2008.

[27] P. S. Sellamuthu, M. Mafune, D. Sivakumar, and P. Soundy, "Thyme oil vapour and modified atmosphere packaging reduce anthracnose incidence and maintain fruit quality in avocado," Journal of the Science of Food and Agriculture, vol. 93, no. 12, pp. 3024-3031, 2013.

[28] A. Ali, M. Maqbool, P. G. Alderson, and N. Zahid, "Effect of gum Arabic as an edible coating on antioxidant capacity of tomato (Solanum lycopersicum L.) fruit during storage," Postharvest Biology and Technology, vol. 76, pp. 119-124, 2013.

[29] G. K. Jayaprakasha, P. S. Negi, B. S. Jena, and L. Jagan Mohan Rao, "Antioxidant and antimutagenic activities of Cinnamomum zeylanicum fruit extracts," Journal of Food Composition and Analysis, vol. 20, no. 3-4, pp. 330-336, 2007.

[30] M. V. Martinez and J. R. Whitaker, "The biochemistry and control of enzymatic browning," Trends in Food Science \& Technology, vol. 6, no. 6, pp. 195-200, 1995.

[31] K. Tano, M. K. Oulé, G. Doyon, R. W. Lencki, and J. Arul, "Comparative evaluation of the effect of storage temperature fluctuation on modified atmosphere packages of selected fruit and vegetables," Postharvest Biology and Technology, vol. 46, no. 3, pp. 212-221, 2007.

[32] A. A. Kader, "Prevention of ripening in fruits by use of controlled atmospheres," Food Technology, vol. 34, pp. 51-54, 1980.

[33] X. Duan, X. Su, Y. You, H. Qu, Y. Li, and Y. Jiang, "Effect of nitric oxide on pericarp browning of harvested longan fruit in relation to phenolic metabolism," Food Chemistry, vol. 104, no. 2, pp. 571-576, 2007.

[34] V. Chiabrando and G. Giacalone, "Effect of essential oils incorporated into an alginate-based edible coating on freshcut apple quality during storage," Quality Assurance and Safety of Crops \& Foods, vol. 7, no. 3, pp. 251-259, 2015.

[35] G. A. Ojeda, S. C. Sgroppo, and N. E. Zaritzky, "Application of edible coatings in minimally processed sweet potatoes (Ipomoea batatas L.) to prevent enzymatic browning," International Journal of Food Science \& Technology, vol. 49, no. 3, pp. 876-883, 2014.

[36] M. Gao, L. Feng, and T. Jiang, "Browning inhibition and quality preservation of button mushroom (Agaricus bisporus) by essential oils fumigation treatment," Food Chemistry, vol. 149, pp. 107-113, 2014

[37] P. Jin, X. Wu, F. Xu, X. Wang, J. Wang, and Y. Zheng, "Enhancing antioxidant capacity and reducing decay of Chinese bayberries by essential oils," Journal of Agricultural and Food Chemistry, vol. 60, no. 14, pp. 3769-3775, 2012. 